Pesq. Vet. Bras. 28(4):201-206, abril 2008

\title{
Avaliação do uso de haste bloqueada e bloqueio transcortical no reparo de fraturas diafisárias de fêmur em felinos ${ }^{1}$
}

\author{
Leandro Romano ${ }^{2 *}$, Cássio R. Auada Ferrigno ${ }^{3}$ Vanessa C. Magalhães \\ Ferraz ${ }^{2}$, Marcos Ishimoto Della Nina ${ }^{2}$ e Kelly Cristiane Ito ${ }^{2}$
}

\begin{abstract}
Romano L., Ferrígno C.R.A., Ferraz,V.C.M. Della Nina M., Ito K.C. 2008. [Evaluation of the use of interlocking nail and transcortical blockade for the repair of diaphyseal fractures of the femur in cats.] Avaliação do uso de haste bloqueada e bloqueio transcortical no reparo de fraturas diafisárias de fêmur em felinos. Pesquisa Veterinária Brasileira 28(4):201-206. Departamento de Cirurgia do Hospital Veterinário, Faculdade de Medicina Veterinária e Zootecnia, Universidade de São Paulo, Avenida Prof. Dr. Orlando Marques de Paiva 87, São Paulo, SP 05508-270, Brazil. E-mail: Iromano@usp.br

Interlocking nails allow rigid fracture stabilization and present biomechanical advantages when compared to other immobilization techniques. It acts along the central mechanical axis of the bone and preserves biological concept standards for osteosynthesis. The use of interlocking nail in cats is limited because the intramedullary space is relatively small for the available nail sizes. We reported 10 cases of cats that had simple or multiple closed fractures of the femoral diaphysis. The animals weighed 3.5 to $5 \mathrm{~kg}$, which allowed using nails of $4.0 \mathrm{~mm}$ and $4.7 \mathrm{~mm}$ in diameter. For the nail insertion, retrograde and normograde routs were used, and four $2 \mathrm{~mm}$ titanium screws were used to lock all the perforations. No trans-surgical complication occurred, however, due to a new trauma, one of the animals suffered femoral neck fracture and transversal distal fracture to the nail, three days after surgery. Radiographies were made up to four months after surgery, and in most cases secondary bone formation was seen, showing bone consolidation in 61-89 days, excluding the animal who suffered new trauma, who presented bone healing in 150 days. Clinically, occasional lameness occurred; however, except for the animal that had new trauma, all animals initiated adequate limb support in 3 to 8 days after surgery. In conclusion, the use of $4.0 \mathrm{~mm}$ and $4.7 \mathrm{~mm}$ interlocking nails in felines was adequate, with bone healing in all cases, and also return of good limb function after surgery.
\end{abstract}

INDEX TERMS: Fractures, interlocking nail, surgery, cats.

RESUMO.- As hastes bloqueadas além de permitirem estabilização rígida de fraturas, possuem vantagens biomecânicas quando comparadas a outras técnicas de imobilização, por atuar ao longo do eixo mecânico central

\footnotetext{
${ }^{1}$ Recebido em 19 de junho de 2007.

Aceito para publicação em 17 de março de 2008.

2 Departamento de Cirurgia, Hospital Veterinário, Faculdade de Medicina Veterinária e Zootecnia (FMVZ), Universidade de São Paulo (USP), Av. Prof. Dr. Orlando Marques de Paiva 87, Cidade Universitária, São Paulo, SP 05508-270, Brasil. *Autor para correspondência: Iromano@usp.br

${ }^{3}$ Pós-Graduando, Departamento de Cirurgia, FMVZ, USP, São Paulo, Brasil.
}

do osso, além de preservar os conceitos de padrões biológicos de osteossíntese. O uso limitado de hastes bloqueadas em gatos se atribui ao fato da cavidade intramedular ser pequena, limitando o tamanho e diâmetro dos pinos. Relatam-se casos de 10 felinos que apresentavam fraturas fechadas simples ou múltiplas da diáfise femoral. Os animais pesavam entre 3,5 e $5 \mathrm{~kg}$, o que permitiu a utilização de hastes de 4,0mm e 4,7mm de diâmetro. Para inserção da haste, adotou-se a via retrógrada ou a normógrada e foram realizados bloqueios com quatro parafusos de titânio de $2,0 \mathrm{~mm}$ de diâmetro. Complicações intra-operatórias não ocorreram, entretanto um animal sofreu fratura de colo femoral e fratura transversa distal 
ao implante, três dias após o ato cirúrgico, devido a novo trauma. Os animais foram submetidos a exames radiográficos até quatro meses após a intervenção, evidenciando-se formação de calo ósseo secundário e consolidação óssea em período de 61-89 dias. Clinicamente, ocorreu esporadicamente alteração na marcha por leve claudicação de apoio definida por escala de claudicação, entretanto os animais iniciaram o apoio adequado entre três e oito dias após o ato operatório. Complicações pósoperatórias ocorreram referentes ao animal que sofreu novo trauma, entretanto notou-se consolidação do foco primário tratado pelo implante com 61 dias e consolidação da nova fratura num período total de 150 dias do início do tratamento conservativo por penso esparadrapado e tala. Conclui-se que o uso de haste bloqueada em felinos foi adequado uma vez que houve consolidação óssea em todos os casos, com retorno precoce da função do membro, permitindo a deambulação.

TERMOS DE INDEXAÇÃO: Fraturas, hastes bloqueadas, cirurgia, felinos.

\section{INTRODUÇÃO}

A fixação intramedular como método de estabilização de fraturas é conhecida e utilizada desde o século XVI, contudo a técnica de hastes bloqueadas foi primeiramente descrita em pacientes humanos no princípio dos anos de 1950, por Modney (Aron 1995).

Fraturas de ossos longos são um dos principais pontos de tratamento ortopédico na medicina veterinária. Estas fraturas usualmente ocorrem devido às injúrias de alto impacto, quedas de locais altos, atropelamentos e feridas por projéteis balísticos. (Larin et al. 2001).

O uso de hastes bloqueadas, além de prevenir os movimentos responsáveis pelo desencadeamento do processo de não-união óssea é biomecanicamente vantajoso em relação à outras técnicas de imobilização, por atuar ao longo do eixo mecânico central do osso (Moses et al. 2002), além de preservar os conceitos de padrões biológicos de osteossíntese (Duhautois 2003).

O conceito de recuperação biológica das fraturas foi introduzido há alguns anos, no intuito de preservar, o máximo possível, a integridade vascular dos fragmentos ósseos e ao mesmo tempo, prover fixação capaz de manter o alinhamento e o comprimento ósseo durante o período de reparação. O alinhamento dos fragmentos ósseos maiores e das articulações predomina sobre a reconstrução anatômica (Woo et al. 1984, Perren 1991, Aron et al. 1995, Berardé et al. 2002). Em medicina veterinária, instituiu-se técnica que consiste em mínimas incisões, com o menor contato possível com os tecidos moles adjacentes ao foco de fratura e que preserva o potencial osteogênico do hematoma provocado pela fratura (McLaughlin 1999, Palmer 1999, Bernarde et al. 2001, Horstman et al. 2004), a vascularização, reduz o tempo cirúrgico e minimiza a possibilidade de infecção pós-operatória (McClure, et al. 1998, Lopez et al. 2001, Horstman et al. 2004).
A escolha correta dos implantes se dá pela relação entre o menor diâmetro no espaço intramedular e o comprimento do osso, sendo definido pelo uso de um filme de transparência, impresso com a forma da haste além da discrepância de aumento que se dá no filme radiográfico. Após alocar o filme sobre a radiografia do membro contralateral íntegro, define-se o tamanho ideal do pino. (Schmaedecke 2007)

O uso limitado de hastes bloqueadas em gatos se atribui ao fato da cavidade intramedular ser pequena restringindo o tamanho, que varia entre $0,68 \mathrm{~mm}$ e $134 \mathrm{~mm}$, e o diâmetro, de $4 \mathrm{~mm}$ e 4,7mm, dos pinos. (Larin 2001).

A proposta deste estudo foi de avaliar o tempo de consolidação óssea com o uso de hastes bloqueadas em felinos, as variações existentes entre os tipos de calo durante o processo de reparação da fratura e possíveis complicações.

\section{MATERIAL E MÉTODOS}

Foram utilizados pacientes da espécie felina, trazidos ao Hospital Veterinário de Universidade de São Paulo para atendimento e foram encaminhados ao Laboratório de Ortopedia (LOTC). Os animais apresentavam fraturas fechadas, simples ou múltiplas, da diáfise femoral e pesavam entre 3,5 e 5,0 kg, o que permitiu somente a utilização de hastes de 4,0 e $4,7 \mathrm{~mm}$ de diâmetro.

Adotou-se como padrão exame radiográfico tanto da região fraturada quanto do membro contralateral durante o atendimento inicial. Com auxílio de filme de transparência padrão que acompanha o instrumental de hastes bloqueadas, alocado por sobre a radiografia do membro íntegro, foram definidos os tamanhos ideais de pinos a serem utilizados no membro fraturado em cada caso (Fig.1 e 4).

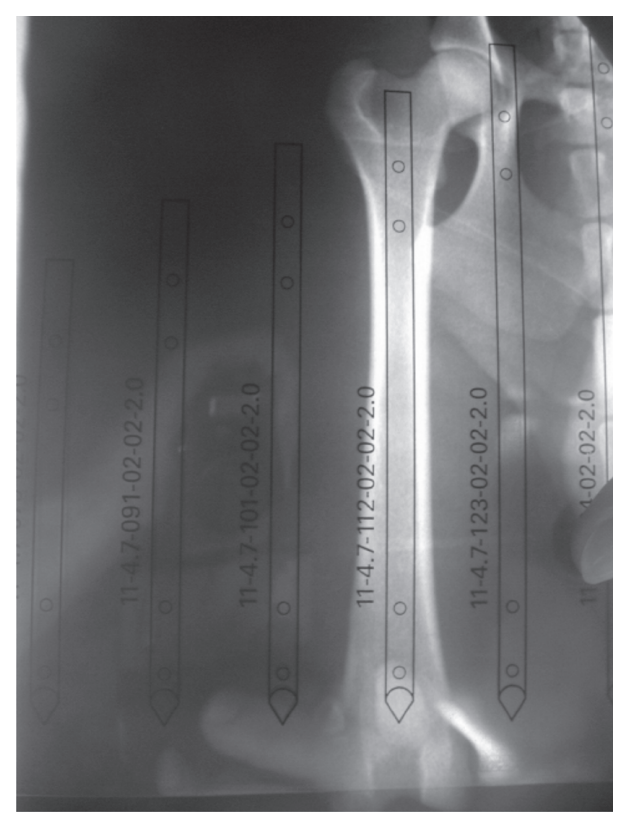

Fig.1. "Template" padrão feito em filme de transparência alocado por sobre a radiografia de fêmur em posição crânio-caudal do membro contralateral do animal $n^{\circ} 6$ para definição dos tamanho ideal do pino. 

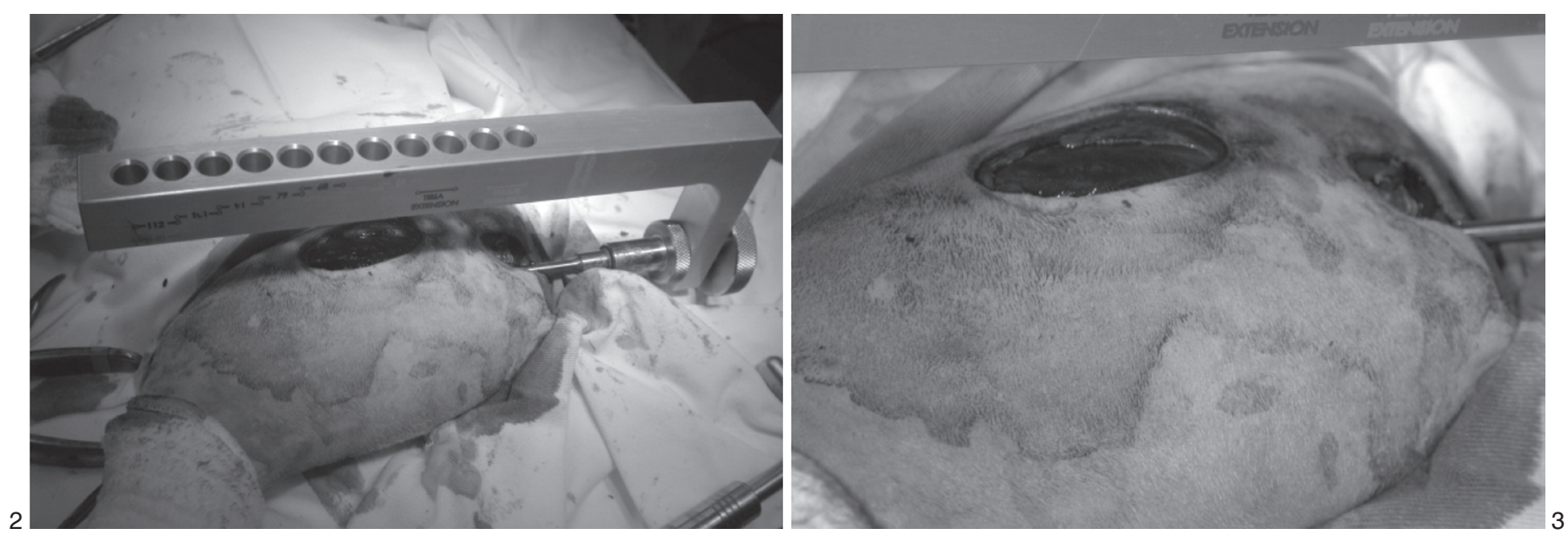

Fig.2 e 3. Observar dois pequenos acessos, um de cerca de $4 \mathrm{~cm}$ para redução da fratura e outro de aproximadamente $2 \mathrm{~cm}$ para posicionamento da haste (Animal $n^{\circ}$ 6).

Após escolha do implante, os felinos foram anestesiados, conforme protocolo do laboratório. Após tricotomia e antissepsia da região os dois acessos foram realizados por incisão de pele, tecido celular subcutâneo. Para o acesso dorsal abordou-se a região do trocânter maior e exposição da fossa intertrocantérica. Realizou-se acesso lateral e divulsão do músculo bíceps femoral rebatido caudalmente, músculo vasto lateral e fascia lata cranialmente, expondo assim a região da fratura para redução. (Fig.2 e 3).

$\mathrm{O}$ osso foi previamente fresado, para que nenhuma força adicional fosse necessária durante a introdução da haste. Para inserção da haste, foram adotadas as vias normógrada ou retrógrada e foram realizados bloqueios com três ou quatro parafusos de titânio de 2,0mm de diâmetro. Em relação aos bloqueios, utilizaram-se as marcações específicas do guia, para o ta-

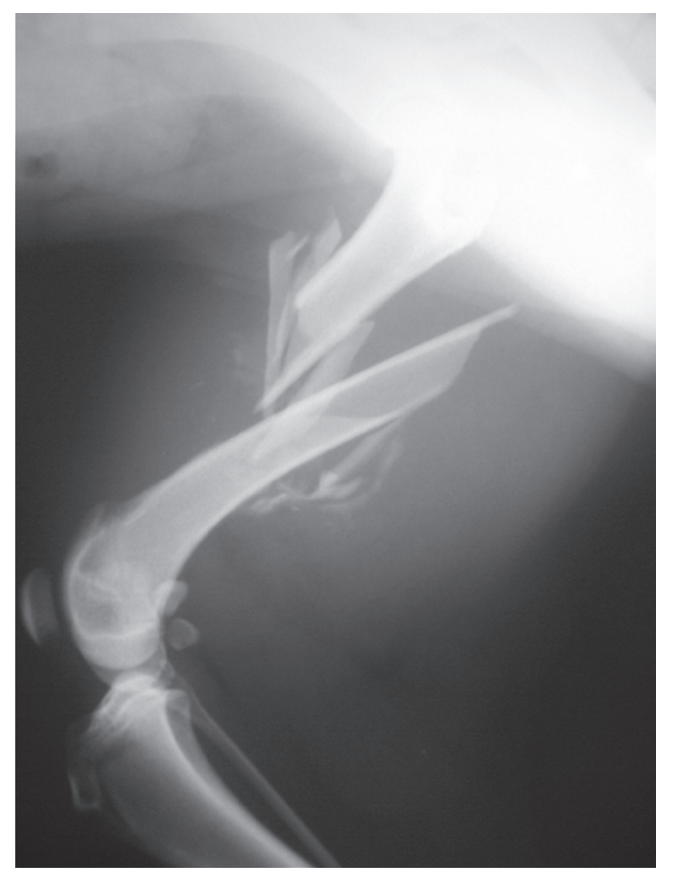

Fig.4. Imagem radiográfica em posição latero-lateral do animal $\mathrm{n}^{\circ} 6$, revelando fratura cominutiva em diáfise femural.

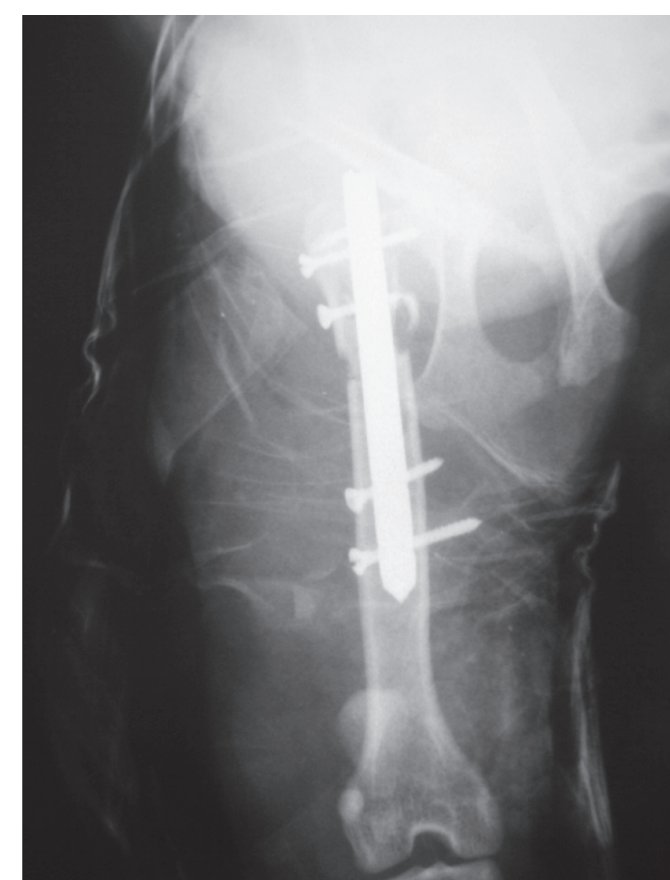

Fig.5. Imagem radiográfica de 7 dias de pós-operatório. Notar o posicionamento da haste e parafusos.

manho do pino escolhido (Fig.3). O orifício foi realizado através das duas corticais, com a broca alcançando a cortical distal por transposição do orifício existente na haste. As duas corticais foram então machadas, e o parafuso fixado, bloqueando a haste (Fig.5).

Os animais foram submetidos a exames radiográficos no pós-operatório imediato certificação do posicionamento da haste e a cada 30 dias para acompanhamento do processo de consolidação.

O grau de claudicação no pós-operatório foi realizado através de escores, por avaliação temporal e marcha, onde: 0 = apoiava o membro normalmente; 1 = claudicação leve com retração esporádica do membro; 2 = claudicação moderada com apoio durante locomoção lenta e retração durante locomoção rápida; 3 = Claudicação grave com apoio apenas durante estação; 4 = 

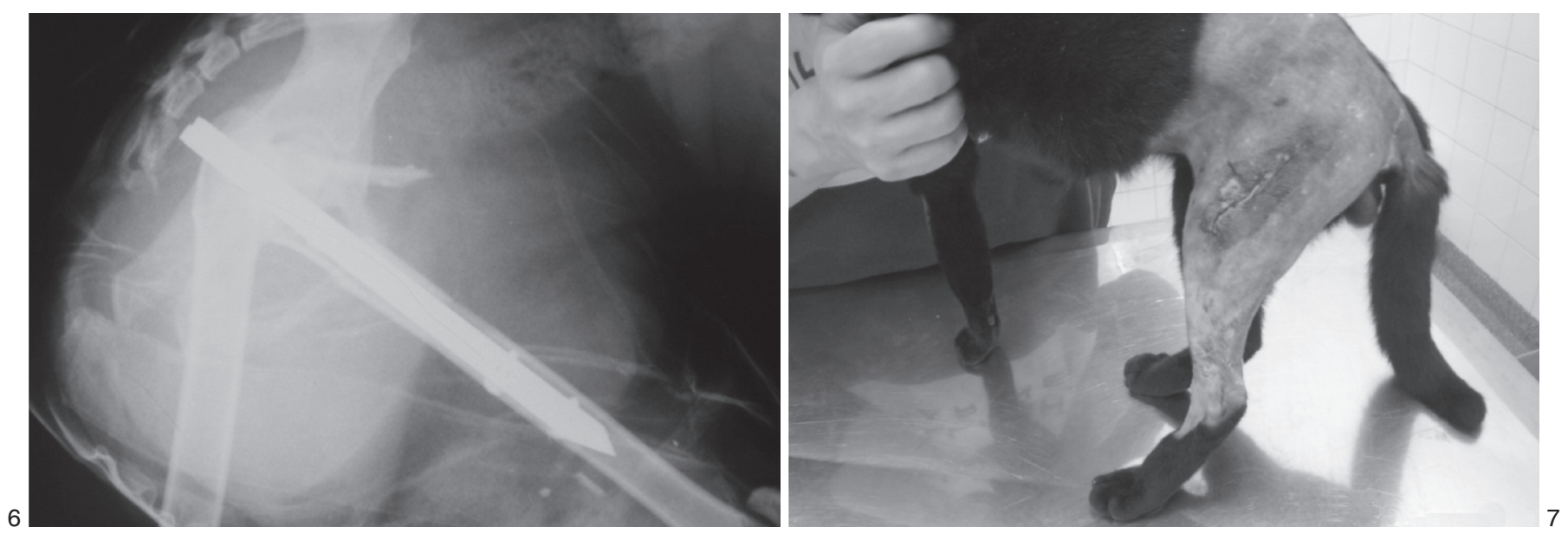

Fig.6. Calo ósseo evidente com 64 dias em imagem radiográfica de fêmur em posição latero-lateral.

Fig 7. Apoio adequado do membro após 7 dias de pós-operatório.

Quadro 1. Resultados obtidos com o uso de haste bloqueada em felinos

\begin{tabular}{|c|c|c|c|c|c|c|c|c|c|}
\hline $\begin{array}{c}\text { Animal } \\
\text { no. }\end{array}$ & $\begin{array}{l}\text { Raça }{ }^{a} \\
\text { (anos) }\end{array}$ & Idade & Sexob & $\begin{array}{l}\text { Tamanho do } \\
\text { implante } \\
(\mathrm{mm})\end{array}$ & $\begin{array}{l}\text { Bloqueios } \\
\text { (número) }\end{array}$ & $\begin{array}{l}\text { Tempo de } \\
\text { conso- } \\
\text { lidação } \\
\text { (dias) }\end{array}$ & $\begin{array}{l}\text { Tipo de } \\
\text { calo }\end{array}$ & $\begin{array}{l}\text { Retorno do } \\
\text { membro } \\
\text { à função } \\
\text { (dias) }\end{array}$ & Complicações \\
\hline 1 & Srd & 2anos e 1 mês & $\mathrm{F}$ & 4,0 & 4 & 70 & Secundário & 6 & Nenhuma \\
\hline 2 & Srd & 3 anos e 5 meses & M & 4,0 & 4 & 64 & Secundário & 4 & Nenhuma \\
\hline 3 & Srd & 5 anos e 1 mês & M & 4,0 & 4 & 72 & Secundário & 4 & Nenhuma \\
\hline $4^{\mathrm{C}}$ & Srd & 4 anos e 7 meses & $\mathrm{F}$ & 4,7 & 4 & 61 & Secundário & $\begin{array}{l}30 \text { após } \\
\text { novo } \\
\text { trauma }\end{array}$ & $\begin{array}{l}\text { Fratura de colo } \\
\text { femoral e fratura } \\
\text { transversa distal } \\
\text { ao implante }\end{array}$ \\
\hline 5 & Srd & 2 anos e 3 meses & M & 4,0 & 4 & 80 & Secundário & 5 & Nenhuma \\
\hline 6 & Srd & 5 anos e 8 meses & $\mathrm{F}$ & 4,7 & 4 & 89 & Secundário & 3 & $\begin{array}{l}\text { Alteração esporá- } \\
\text { dica na marcha }\end{array}$ \\
\hline 7 & Srd & 3 anos e 6 meses & M & 4,0 & 4 & 74 & Secundário & 5 & Nenhuma \\
\hline 8 & Srd & 3 anos e 11 meses & M & 4,0 & 3 & 70 & Secundário & 8 & Nenhuma \\
\hline 9 & Srd & 4 anos e 2 meses & M & 4,0 & 4 & 64 & Secundário & 5 & Nenhuma \\
\hline 10 & Srd & 2 anos e 3 meses & M & 4,0 & 4 & 72 & Secundário & 4 & Nenhuma \\
\hline
\end{tabular}

Quadro 2. Resultado temporal do escore de fratura

\begin{tabular}{|c|c|c|c|c|c|c|c|c|c|c|}
\hline Animais & 01 & 02 & 03 & 04 & 05 & 06 & 07 & 08 & 09 & 10 \\
\hline $\begin{array}{l}\text { Escore } \\
7 \text { dias }\end{array}$ & 1 & 2 & 1 & 4 & 2 & 2 & 2 & 1 & 1 & 2 \\
\hline $\begin{array}{l}\text { Escore } \\
30 \text { dias }\end{array}$ & 0 & 1 & 0 & 3 & 1 & 1 & 1 & 0 & 0 & 1 \\
\hline $\begin{array}{l}\text { Escore } \\
60 \text { dias }\end{array}$ & 0 & 0 & 0 & 2 & 0 & 0 & 0 & 0 & 0 & 0 \\
\hline $\begin{array}{l}\text { Escore } \\
90 \text { dias }\end{array}$ & 0 & 0 & 0 & 2 & 0 & 0 & 0 & 0 & 0 & 0 \\
\hline $\begin{array}{c}\text { Escore } \\
120 \text { dias }\end{array}$ & 0 & 0 & 0 & 0 & 0 & 0 & 0 & 0 & 0 & 0 \\
\hline
\end{tabular}

não apoiava o membro (Sprecher 1997, Quin et al. 2007). (Quadro 2).

\section{RESULTADOS}

Obteve-se predominância de animais sem raça definida, entre 2 e 5 anos de idade, sendo sete machos e três fêmeas.

Foram utilizados pinos de $4 \mathrm{~mm}$ e $4,7 \mathrm{~mm}$ de diâmetro, com comprimentos de $0,68 \mathrm{~mm}, 0,79 \mathrm{~mm}, 0,91 \mathrm{~mm}$,
$101 \mathrm{~mm}, 112 \mathrm{~mm}, 123 \mathrm{~mm}$ e $134 \mathrm{~mm}$, apresentando dois furos proximais e dois furos distais para bloqueio com parafusos de 2,0mm. Na maioria dos casos foi obtido bloqueio adequado dos quatro orifícios e em somente um caso foi utilizado bloqueio de três orifícios.

Notou-se formação de calo ósseo secundário em todos os animais em período variável entre 61 e 89 dias, com apoio adequado e retorno do membro à função entre três e oito dias de pós-operatório.

Ocorreu complicação pós-operatória em um animal que sofreu fratura de colo femoral e fratura transversa distal ao implante após três dias da intervenção cirúrgica, devido a novo trauma. A fratura primária foi tratada pelo uso de haste bloqueada sendo detectada a consolidação aos 61 dias de pós-cirúrgico e a fratura secundária foi tratada por meio de penso esparadrapado e tala sendo notada consolidação aos 150 dias após início do tratamento conservativo.

Os animais foram submetidos a exames a cada 30 dias até quatro meses após o ato operatório, revelando consolidação óssea em todos os casos, baseada na pre- 
sença de ponte óssea, e ausência de linha de fratura (Fig.6). O grau de claudicação foi avaliado através de escores conforme tabela abaixo.

A evolução clínica baseada em exame radiográfico, exame físico com início precoce do retorno a função do membro e observação do proprietário foi considerada excelente na maioria dos casos e razoável em um animal que sofreu nova fratura (Fig.7).

\section{DISCUSSÃO}

As hastes bloqueadas são pinos intramedulares com furos transversais posicionados em distâncias padronizadas, atuando ao longo do eixo mecânico central do osso (Larin 2001). O implante usado permitiu por entre os orifícios realizar bloqueio transcortical através de parafusos, anulando os movimentos axiais e rotacionais. O pino foi inserido sem complicações de forma intramedular normógrada ou retrógrada e os parafusos foram fixados em todos orifícios proximal e distalmente à fratura.

A neutralidade da colocação do pino, a geometria dos mesmos ao bloqueio das forças, a fadiga provocada sobre o implante foram adequadas e suficientes para promover consolidação óssea.

Além disso, os pinos de haste bloqueada puderam ser implantados através de acessos cirúrgicos menores quando comparado a outros métodos de tratamento como placas ósseas (McClure et al. 1998, Palmer 1999; Lopez et al. 2001), minimizando a manipulação e incisão de tecidos moles adjacentes, implicando assim na manutenção da integridade biológica do foco da fratura.

Concorda-se com a idéia de que a interface parafusopino é extremamente rígida, entretanto permite micromovimentos que estimulam formação de calo ósseo, e isto foi visto em nosso experimento pela formação de calo ósseo secundário nos casos estudados. (Duhautois 2003).

A indicação da técnica depende das condições ósseas locais, tais como presença de fratura simples, multifragmentada ou cominutiva em diáfise, viabilidade dos fragmentos distal e proximal passíveis da inserção dos parafusos para bloqueio transcortical, possíveis fissuras nestes fragmentos, integridade das articulações adjacentes e como visto no trabalho em questão, as hastes bloqueadas podem ser usadas com sucesso em fraturas que apresentem lesões complexas, devido a menor injuria iatrogênica à vascularização óssea, o mesmo foi relatado por Lopez et al. (2001) e Horstman et al. (2004).

Os objetivos do tratamento com hastes bloqueadas foram eliminar a dor, estabilizar o foco da fratura, evitar atrofia dos grupos musculares envolvidos, permitindo desta forma, o adequado apoio precoce do membro durante a marcha. (McClure et al. 1998, Palmer 1999; Larin 2001, Lopez et al. 2001, Duhautois 2003, Horstman et al. 2004), o que foi atingido na maioria dos casos.

Relacionado a escolha dos implantes, adotou-se o membro contralateal para mensuração do tamanho do pino, se dando pela relação entre o menor diâmetro no espaço intramedular e o comprimento do osso. Notou-se que em alguns casos o comprimento e o diâmetro do canal medular do osso variaram, acarretando em um posicionamento de fixação diferenciado dos parafusos distais, bem como quanto do tamanho do implante, confirmando a idéia de Larin (2001), de que o uso limitado de hastes bloqueadas em gatos se atribui ao fato da cavidade intramedular ser pequena, contudo, não foi fator complicante tanto para aplicação do implante quanto para consolidação óssea.

O uso do filme de transparência permitiu a definição do diâmetro e comprimento da haste, e a discrepância de aumento que se dá no filme radiográfico não foi considerada complicante. (Schmaedecke 2007).

O emprego de haste bloqueada, em todos os casos deste estudo, foi posicionado de forma estática, no reparo de fraturas femorais em felinos, mas como foi demonstrado por Schmaedecke (2007), a diferença do diâmetro entre a haste e o canal medular propiciou pequeno movimento no foco de fratura, o que levou a formação de calo ósseo secundário, este dado também é corroborado pelas publicações da AO (Arbeitsgemeinschaft für Osteosynthesefragen) específicas sobre hastes intramedulares (Roe 2005).

A avaliação do grau de claudicação se deu pelo escore de claudicação por avaliação temporal e marcha, idealizado por Sprecher (1997). Clinicamente na maioria dos animais ocorreu esporádica alteração na marcha por leve claudicação de apoio nos primeiros dias seguindo-se a apoio adequado entre três e oito dias após ato operatório, até o apoio normal. $\mathrm{O}$ animal número quatro obteve escore de claudicação quatro devido ao trauma posterior. Optou-se por tratamento conservativo posterior devido à restrição referente ao proprietário. Foram realizados pensos esparadrapados e talas no intuito de minimizar a dor, edema e forças que impedem o processo de consolidação óssea. Estes pensos foram trocados periodicamente com intervalos máximos de 15 dias, notando-se evolução clínica no que se referia à dor e o retorno do membro à função. O escore alto conferido no início do tratamento foi diminuindo de acordo com a consolidação obtida pelo tratamento conservativo adotado.

Vale ressaltar que o animal possuía, então, duas fraturas distintas tratadas por meios diferentes, uma pelo implante e outra por penso esparadrapado e tala. Evidenciou-se um processo de consolidação no foco primário, num período de 61 dias denotando uma evolução conforme os outros animais do experimento, uma vez que os fragmentos ósseos bloqueados estavam estáveis. Já no foco secundário o tratamento escolhido permitiu formação de calo ósseo secundário exuberante num período de tempo de 150 dias, além de manter o conforto e ausência de dor durante a recuperação do animal.

Relacionado o escore de claudicação conferido a este paciente, nota-se uma discrepância muito grande em relação ao escore dado aos outros pacientes, visto a complicação ocorrida sendo determinante no apoio do animal no pós-operatório. $O$ escore deste paciente não condiz 
com a realidade na reparação de fraturas diafisárias por hastes bloqueadas em felinos, uma vez que obtivemos semelhança nos outros animais.

Notou-se calo ósseo secundário em todas as reparações e tempo de consolidação adequado variando entre 60 e 90 dias, sendo que próximo a 65 dias ocorreu maior evolução na consolidação óssea. Relacionado à deambulação, alguns animais apresentaram alteração esporádica na marcha caracterizado por claudicação de apoio leve, não sendo considerada relevante no processo de consolidação.

Em todos os animais optou-se pela manutenção dos implantes após a completa consolidação óssea, uma vez que até o momento não encontramos halos de osteólise ou aumento de volume na região dos parafusos, osteólise ao redor da haste, quebra dos implantes ou dor. Sendo assim, em não havendo desconforto de nenhum paciente os implantes foram mantidos.

Com base em nossos resultados, o emprego de haste bloqueada foi vantajoso no tratamento de pacientes com fratura diafisária de alta complexidade. Este método de fixação permitiu o alinhamento ósseo ideal, possibilitou a correção e bloqueio dos desvios rotacionais, de cisalhamento, de tração e compressão, dispensou o uso de penso esparadrapado por tempo prolongado de maneira a evitar atrofia dos grupos musculares envolvidos e obteve-se fixação óssea estável, culminando na formação de calo ósseo secundário e apoio precoce do membro operado.

Tal técnica se mostra clinicamente efetiva, pois respeita os padrões biológicos de tratamento de fraturas (Woo et al. 1984, Perren 1991, Aron et al. 1995, Berardé et al. 2002) e ainda assim confere estabilidade ao osso, permitiu reabilitação adequada com baixos índices de complicações, propiciando assim as condições necessárias para a consolidação óssea.

\section{CONCLUSÕES}

Conclui-se, então, que o uso de haste bloqueada em felinos é técnica factível, e nos casos estudados levou a consolidação óssea, com retorno precoce da função do membro, permitindo a deambulação.

\section{REFERENCIAS}

Aron D.N., Palmer R.H. \& Johnson A.L. 1995. Biologic strategies and a balanced concept for repair of highly comminuted long bone fractures. Vet. Comp. Orthop. Traumatol. 7:35-38.

Bernardé A., Diop A., Maurel N. \& Viguier E. 2002. An in vitro biomechanical comparison between bone plate and interlocking nail. 3-D interfragmentary motion and bone strain analysis in ostectomized canine femurs. Vet. Comp. Orthop. Traumatol. 15:57-66.

Duhautois B. 2003. Use of veterinary Interlocking Nails for diaphyseal fractures in dogs and cats: 121 cases. Vet. Surg. 32:8-210.

Horstman C.L., Beale B.S. \& Conzemius M.G. 2004. Biological osteosynthesis versus traditional anatomic reconstruction of 20 longbone fractures using an interlocking nail: 1994-2001. Vet. Surg. 33:232237.

Larin A., Eich C.S., Parker R.B. \& Stubbs W.P. 2001. Repair of diaphyseal femoral fractures in cats using interlocking intramedullary nails: 12 cases (1996-2000). J. Am. Vet. Med. Assoc. 219(8):1098-1104.

McClure S.R., Watkins J.P. \& Ashman R.B. 1998. In vivo evaluation of intramedullary interlocking nail fixation of transverse femoral osteotomies in foals. Vet. Surg. 27:29-36.

McLaughlin R. 1999. Internal fixation, intramedullary pins, cerclagem wires and interlocking nails. Vet. Clin. North Am. Small Anim. Pract. 29:1097-1116.

Moses P.A., Lewis D.D., Lanz O.I., Stubbs W.P., Cross A.R. \& Smith K.R. 2002. Intramedullary interlocking nail stabilization of 21 humeral fractures in 19 dogs and one cat. Aust. Vet. J. 80(6):326-343.

Palmer R.H. 1999. Biological osteosynthesis. Vet. Clin. North Am. Small Anim. Pract. 29:1171-1185.

Perren S.M. 1991. The concept of biological plating using the limited contact: dynamic compression plate (LC-DCP): scientific background, design and application. Injury 22:1-41.

Quinn M.M., Keuler N.S., Lu Y., Faria M. L. E. \& Muir P. 2007. Evaluation of agreement between numerical rating scales, visual analogue scoring scales, and force plate gait analysis in dogs. Vet. Surg. 36(4):360.

Roe S.C. 2005. External fixators, pins, nails, and wires, p.52-72. In: Johnson A.L., Houlton J.E.F. \& Vannini R. (ed.), AO Principles of Fracture Management in the Dog and Cat. AO Thieme Medical Pub.

Schmaedecke A. \& Ferrigno C.R.A. 2007. Avaliação biomecânica do bloqueio transcortical dos implantes de interlocking nail em relação às forças de torção e axiais atuantes em fraturas diafisárias de fêmur de cães: estudo in vitro. Tese de Doutorado, Faculdade de Medicina Veterinária e Zootecnia, USP, São Paulo.

Sprecher D.J., Hostetler D.E. \& Kaneene J.B. 1997. A lameness scoring system that uses posture and gait to predict dairy cattle reproductive performance. Theriogenology 47:1179-1187. 